\title{
Risk of stroke associated with nonsteroidal anti-inflammatory drugs
}

REVIEW

This article was published in the following Dove Press journal:

Vascular Health and Risk Management

6 January 2014

Number of times this article has been viewed

Ki Park

\section{Anthony A Bavry}

Division of Cardiovascular Medicine, University of Florida, Gainesville, FL, USA

Video abstract

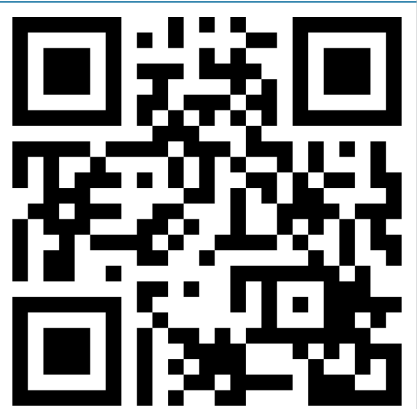

Point your SmartPhone at the code above. If you have a QR code reader the video abstract will appear. Or use: http://dvpr.es/IclrlVT
Correspondence: Anthony A Bavry

Division of Cardiovascular

Medicine, University of Florida,

1600 SW Archer Road, Box 100277,

Gainesville, FL 32610, USA

Email bavryaa@medicine.ufl.edu
Abstract: Nonsteroidal anti-inflammatory drugs (NSAIDs), both cyclooxygenase (COX)2 -selective and nonselective agents, have been associated with the increased risk of adverse cardiovascular events. The majority of studies have focused on myocardial infarction as the primary cardiovascular outcome. However, the association between NSAIDs and the risk of stroke events is not as clear, although an understanding of this association is important since stroke continues to be a significant cause of morbidity and mortality. Various factors may contribute to an association between NSAIDs and stroke, including hypertension and thrombosis. Additionally, the risk may vary with different NSAID types. In this review, we discuss the relevant literature assessing the possible association between NSAID use and stroke events, along with the potential mechanisms and the possible directions for future study.

Keywords: nonsteroidal anti-inflammatory drugs, cardiovascular, stroke, cyclooxygenase, adverse events

\section{Prevalence and risk factors for stroke}

Stroke remains a common cause of morbidity and mortality within the general population. Each year, nearly 800,000 new or recurrent stroke events occur and account for approximately one out of every 18 deaths in the United States. ${ }^{1}$ As the burden of stroke remains high, it is important to identify the factors that may contribute to an increase in risk, especially those factors that may be avoidable. The traditional risk factors are similar to those for myocardial infarction (MI) and include hypertension, diabetes, smoking, and dyslipidemia. ${ }^{1}$ Another nontraditional factor that may contribute to stroke risk is the use of nonsteroidal anti-inflammatory drugs (NSAIDs). NSAIDs are a class of analgesics widely available both in prescription form and over the counter. Much publicity has focused on the adverse cardiovascular effects related to this class of medications; however, the predominant focus has been primarily on the risk of MI. Stroke comprises another important subset of cardiovascular events. Here, we discuss the evidence with regard to an association between selective and nonselective NSAIDs and stroke risk, along with the potential pathophysiologic mechanisms and the targets for future study.

\section{Background}

NSAIDs are a widely available and frequently prescribed class of analgesics. They are among the most commonly purchased or prescribed drugs around the world, used by approximately 30 million people daily. ${ }^{2}$ In the United States, over a million prescriptions are written for NSAIDs every year. ${ }^{3,4}$ Due to concerns over gastrointestinal toxicity, selective COX-2 inhibitors were developed that aimed to eliminate the COX-1-asso- 
ciated adverse effects on the gastric mucosa. However, soon after the introduction of these selective COX-2 inhibitors, red flags started to arise. Withdrawals of several COX inhibitors were based on studies that demonstrated a significantly increased risk of adverse cardiovascular events with use of these COX-2-selective agents. ${ }^{4}$ After the withdrawal of these agents, there was a significant decrease in the prescription of COX-2-selective agents, with a concurrent increase in the prescription of nonselective agents by nearly $20 \% .{ }^{5}$ Currently, ibuprofen is among the most widely used of the over-thecounter NSAIDs in the United States. ${ }^{6}$ Thus, although two COX-2-selective agents were taken off the market in the early 2000s, the prevalence of nonselective NSAID use remains high.

After the withdrawal of rofecoxib and valdecoxib, it soon became evident in subsequent studies that the cardiovascular risk may not only be limited to selective COX-2 inhibitors but also, to nonselective NSAIDs. ${ }^{7,8}$ The majority of the analyses of adverse cardiovascular events have focused on MI or a composite cardiovascular outcome of MI, stroke, and/or heart failure. Despite published American Heart Association guidelines that warn against the use of NSAIDs, and the addition of a black box warning for the NSAID celecoxib, ${ }^{7}$ the results of these studies have led to a generalization of risk across diverse cardiovascular events. However, while the risk of $\mathrm{MI}$ is the most strongly supported by the literature, limited work has focused on an association between NSAID use and risk of other vascular events, specifically stroke. Although the absolute incidence of cerebrovascular events with NSAIDs, compared with MI, is unclear, the importance of any association between NSAID use and strokes should not be overlooked. Thus, a critical review of the literature with regard to specific risk of stroke events is warranted.

\section{Potential mechanisms of NSAID related risk of stroke}

To understand the mechanism of NSAID-induced vascular events, it is important to review the mechanisms by which NSAIDs produce their therapeutic effects. These agents affect the balance of various prostaglandins, thromboxane, and prostacyclin, and their action on vascular function, platelet aggregation, and smooth muscle proliferation. ${ }^{8}$ NSAIDs can generally be differentiated by their level of selectivity for COX, specifically, with the primary isoforms of this enzyme designated as COX-1 and COX-2. COX-1 is ubiquitous in most tissues, while COX-2 is primarily upregulated in inflammatory states. ${ }^{9} \mathrm{COX}$ is responsible for the conversion of arachidonic acid to either thromboxane (mediated by
COX-1) or prostacyclin (mediated by COX-2) (Figure 1). In this review, we classify "selective COX-2 inhibitors" as those agents whose activity is primarily on COX-2. Those NSAIDs that affect both COX-1 and COX-2 in varying degrees are referred to as nonselective agents, and their relative $\mathrm{COX}-1$ and COX-2 activity is represented in Figure $2 .{ }^{10}$ Aspirin is a special NSAID, with primary selective COX-1 inhibition, and its use in secondary prevention of cardiovascular events is established. ${ }^{11}$ Thromboxane is a potent vasoconstrictor, ${ }^{12}$ and its inhibition by aspirin (mediated by COX-1) may be the mechanism for the reduction of vascular adverse events. Prostacyclin is the primary product of COX-2 and is responsible for vasodilation, inhibition of smooth muscle cell proliferation, and platelet inhibition. ${ }^{13}$ Although the inhibition of COX-1 is beneficial from a cardiovascular standpoint, this also affects the gastrointestinal side effect profile, thus, selective COX-2 inhibitors were developed to maintain analgesic efficacy without the disruption of the protective gastrointestinal mucosal effects mediated by COX-1. It has been proposed from the literature that an imbalance between thromboxane and prostacyclin can influence the relative adverse vascular effects of NSAIDs (Table 1). ${ }^{13}$ Although the imbalance of relative COX-2 to COX-1 effects is the most widely propagated theory for the harmful effects of NSAIDs, emerging data suggest this concept may not be completely accurate for a variety of reasons. Foremost, there has been significant variability in the determination of COX selectivity by different assay methodologies. ${ }^{14}$ This complicates any general conclusions regarding a linear relationship between relative COX-2 selectivity and adverse vascular events. Additionally, the concept that only COX-2 is expressed in the endothelium (driving endothelial adverse effects) has been recently challenged. ${ }^{15}$ Thus, other mechanisms, such as NSAID-induced alterations in aldosterone metabolism, ${ }^{16}$

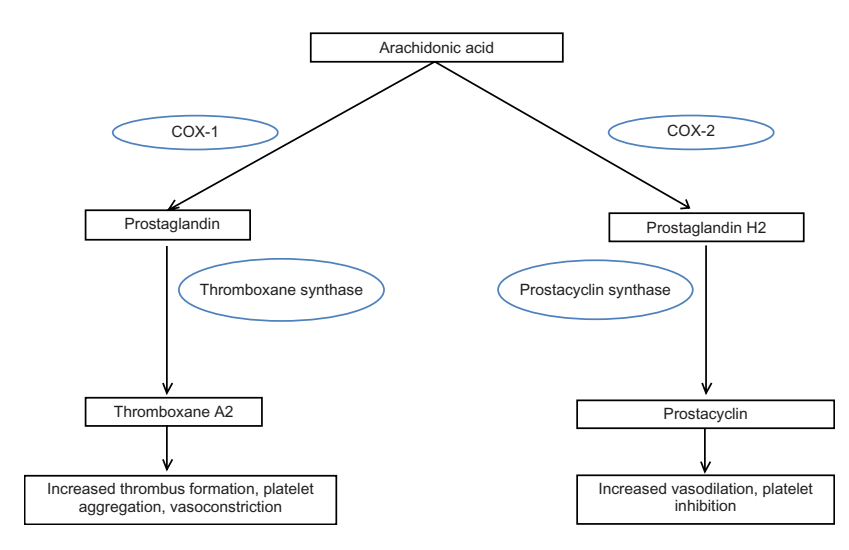

Figure I Effects of COX inhibition on vasculature. Abbreviation: COX, cyclooxygenase. 


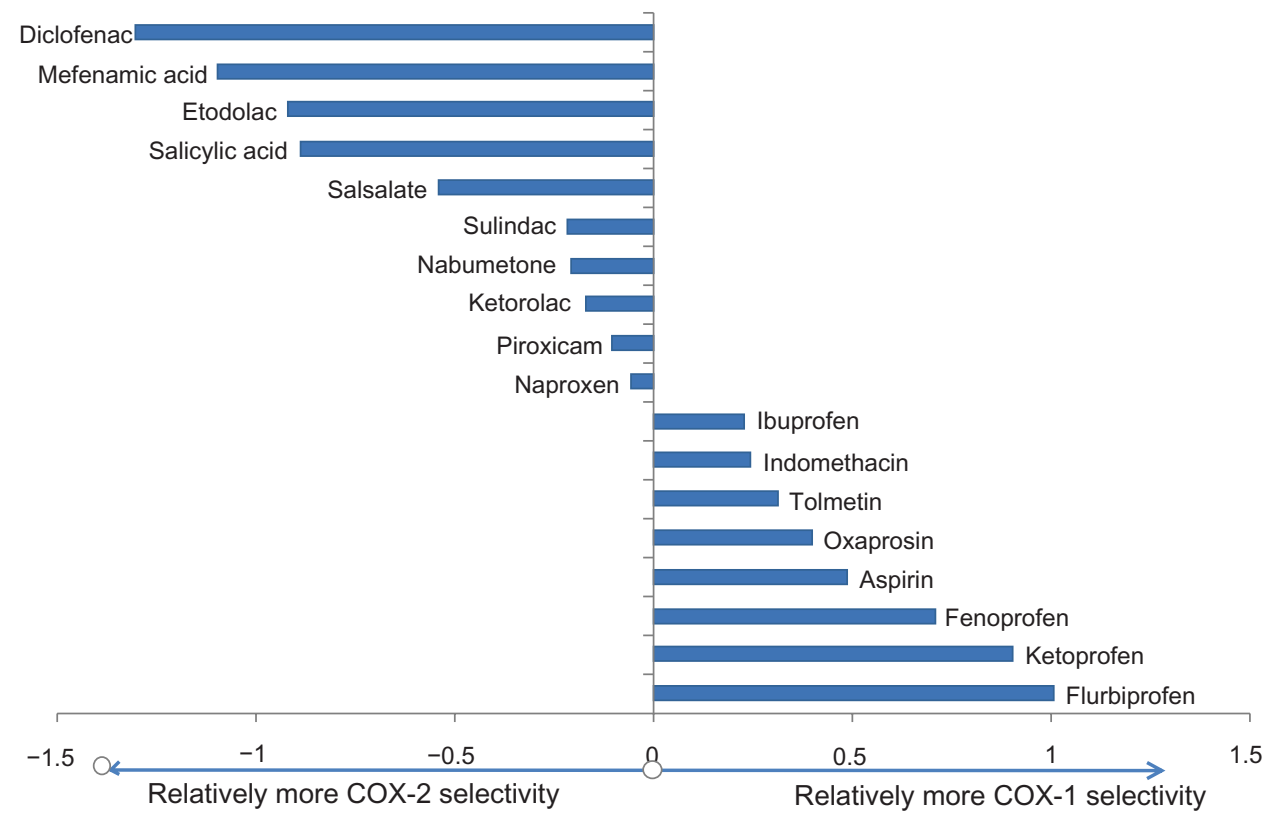

Figure 2 Relative COX-1/COX-2 selectivity of various nonselective NSAIDs.

Note: This chart represents the log $\left(I_{50}\right.$ of COX-I relative to the $I_{50}$ of COX-2) for various nonselective NSAIDs. Adapted with permission Cryer B, Feldman M. Cyclooxygenase-I and cyclooxygenase-2 selectivity of widely used nonsteroidal anti-inflammatory drugs. Am J Med. 1998; I04(5):4I3-42I. ${ }^{0}$ CC Copyright I 998, with permission Elsevier.

Abbreviations: COX, cyclooxygenase; $I_{50}$, half maximal inhibitory concentration; NSAID, nonsteroidal anti-inflammatory drug.

may also be involved, and this highlights the need for further study in this area.

All NSAIDs, to some degree, affect vasoconstriction and sodium excretion, which can lead to hypertension, a risk factor for cerebrovascular events. ${ }^{17}$ Among the nonselective agents, studies have shown naproxen to be associated with the highest increase in blood pressure. ${ }^{18-22}$ Among the COX-2-selective agents, studies ${ }^{4,23,24}$ have demonstrated the blood pressure elevations with COX-2-selective agents to be highest with rofecoxib. Although studies have suggested an association between blood pressure increase and NSAID use, the focus was primarily on short-term duration with use less than 30 days. ${ }^{22,25}$ In a study among hypertensive patients with coronary disease, it was found that chronic NSAID use for close to 3 years was actually associated with slightly lower blood pressure levels compared with nonuse. ${ }^{26}$ Thus, although a causal association between NSAID use and stroke risk is plausible based on their vascular effects, any hypertension-mediated effect

Table I Potential mechanisms for NSAID-associated increase in stroke risk

Vasoconstriction secondary to inhibition of prostacyclin-induced vasodilation

Hypertension induced by direct renal effects on sodium excretion leading to volume expansion

Thrombosis due to prostaglandin-mediated platelet aggregation

Abbreviation: NSAID, nonsteroidal anti-inflammatory drug. may be specific to the duration of NSAID use and warrants further investigation.

\section{Review of study literature}

Much of the initial suggestions of an association between NSAIDs and cardiovascular risk came from prospective studies ${ }^{4,27}$ that were not specifically designed to assess adverse vascular events. The majority of these studies focused primarily on comparing the efficacy of various NSAIDs with regard to prevention of colon adenomas, prevention of gastrointestinal side effects, or the prevention of dementia. In these early studies, which led to the withdrawal of rofecoxib and valdecoxib, the risk of cardiovascular events, particularly MI, was readily apparent. However, the absolute incidence of stroke events compared with coronary events was significantly less, thus making interpretation of any analyses difficult. Within these studies, there was a suggestion of an association between NSAIDs, particularly with rofecoxib, and stroke, although the results were not consistent. The APPROVe trial, in 2005, documented relatively few stroke events and demonstrated a trend toward increased stroke risk with rofecoxib, but this did not reach statistical significance. ${ }^{4}$ The APC (Adenoma Prevention with Celecoxib) trial assessed the use of either $400 \mathrm{mg}$ or $800 \mathrm{mg}$ of celecoxib for the prevention of colorectal adenomas..$^{27} \mathrm{~A}$ composite cardiovascular endpoint was used, thus a specific analysis for stroke was not performed. At $0.4 \%$, the incidence of stroke 
in the low-dose users of celecoxib was not different from placebo, although the incidence was $0.7 \%$ with the higher dose of celecoxib. This same higher dose of celecoxib was actually associated with fewer stroke events as compared with either diclofenac or ibuprofen in another study. ${ }^{28}$ Other subanalyses demonstrated no firm association between stroke risk and the use of naproxen, celecoxib, or rofecoxib. ${ }^{18,29}$

\section{Observational studies}

As the stroke event rate within the previously mentioned studies of NSAIDs for dementia and cancer prevention was insufficient to perform detailed analyses, much of the data specifically demonstrating an association between NSAID use and stroke events are based on observational studies. ${ }^{30-34}$ Although some studies have suggested an association between NSAIDs and stroke risk, these data have not been conclusive; the relevant risk estimates from these studies are listed in Table 2 . An early study performed in 2003 by Bak et al, of over 4,000 patients, demonstrated no increase in the risk of either hemorrhagic or ischemic stroke with any NSAID. ${ }^{30}$ However, several other studies have demonstrated some degree of risk, although the association between specific NSAIDs has varied. The most consistent signal of elevated risk has been with rofecoxib ${ }^{31-33}$ and valdecoxib. ${ }^{31,34}$ A large longitudinal cohort study of nearly 75,000 patients assessed the risk of MI and ischemic stroke. ${ }^{33}$ The researchers found that only rofecoxib was associated with an increased risk for stroke when compared with non-NSAID users, while naproxen was actually associated with a decreased risk. Another study by Andersohn et $\mathrm{al}^{32}$ of over 450,000 patients, studied in a nested case-control analysis, found that use of rofecoxib

Table 2 Hazard ratios of various NSAIDs from observational studies

\begin{tabular}{|c|c|c|c|}
\hline & $\begin{array}{l}\text { Type of } \\
\text { stroke }\end{array}$ & NSAID type & RR or OR (CI) \\
\hline Bak et $\mathrm{al}^{30}$ & Ischemic & Any & $1.2(1.0-1.4)$ \\
\hline Abraham et $\mathrm{al}^{31}$ & $\begin{array}{l}\text { Ischemic or } \\
\text { hemorrhagic }\end{array}$ & *Highly selective & $1.6(1.2-2.2)$ \\
\hline \multirow[t]{3}{*}{ Andersohn et $\mathrm{al}^{32}$} & Ischemic & Rofecoxib & I.7I (I.33-2.I8) \\
\hline & & Etoricoxib & $2.38(1.10-1.53)$ \\
\hline & & Celecoxib & I.07 (0.79-I.44) \\
\hline \multirow[t]{2}{*}{ Solomon et $\mathrm{al}^{33}$} & Ischemic & Rofecoxib & I.I 5 (I.04-I.26) \\
\hline & & Celecoxib & $1.00(0.92-0.09)$ \\
\hline \multirow[t]{3}{*}{ Roumie et $\mathrm{al}^{34}$} & Ischemic or & Rofecoxib & I.28 (I.06-I.53) \\
\hline & hemorrhagic & Valdecoxib & $1.4 \mid(1.04-1.91)$ \\
\hline & & Celecoxib & $1.04(0.87-1.23)$ \\
\hline Haag et $\mathrm{al}^{35}$ & $\begin{array}{l}\text { Ischemic or } \\
\text { hemorrhagic }\end{array}$ & Any & $1.58(1.19-2.08)$ \\
\hline
\end{tabular}

Note: *Highly selective: rofecoxib, valdecoxib, parecoxib and lumiracoxib.

Abbreviations: $\mathrm{Cl}$, confidence interval; NSAID, nonsteroidal anti-inflammatory drug; OR, odds ratio; RR, relative risk. and etoricoxib, but not celecoxib, was associated with an increased risk of ischemic stroke. This study estimated an additional 21 stroke events for rofecoxib and 41 stroke events for etoricoxib per 10,000 exposed persons per year. The risk of stroke has also been suggested to be proportional to the relative degree of COX-2 selectivity. ${ }^{31,35} \mathrm{~A}$ study by Abraham et al in 2007 analyzed over 300,000 patients, categorizing NSAIDs as poorly selective (naproxen and ibuprofen), moderately selective (etodolac, meloxicam, celecoxib, and nabumetone), and highly selective (rofecoxib, etoricoxib, valdecoxib, parecoxib, and lumiracoxib). They found that the incidence of stroke increased proportionately to the degree of COX-2 selectivity and that the use of highly selective agents was significantly associated with stroke compared with poorly selective agents. ${ }^{31}$ Another observational study demonstrated a nearly threefold increase in the risk for ischemic stroke when using selective versus nonselective agents. ${ }^{35}$

\section{Systematic reviews}

As questions remain regarding the association between NSAIDs and the risk of vascular events, several recent metaanalyses have sought to better characterize an association through pooled analyses. The evaluation of these data again demonstrates the paucity of stroke events. In a 2011 network meta-analysis by Trelle et al, of the 31 studies included, 26 provided stroke data, with only 337 observed events. ${ }^{36}$ Interestingly, rofecoxib and celecoxib had the smallest risk estimates for stroke, while ibuprofen and diclofenac were associated with the highest risk. In a recent meta-analysis published by the Coxib and the traditional NSAID Trialists' (CNT) Collaboration in May 2013, the investigators found no association between any NSAID and stroke events when comparing coxibs to either placebo, diclofenac, ibuprofen, or naproxen, although again, relatively few stroke events occurred. ${ }^{37}$ A large meta-analysis of COX-2 inhibitors by Chen and Ashcroft also demonstrated no increase in stroke risk in over 85,000 patients from 40 trials that included comparisons with either placebo or nonselective NSAIDs. ${ }^{38}$ Another meta-analysis limited to analyzing only stroke risk found rofecoxib and diclofenac to have the highest risk of ischemic stroke. ${ }^{39}$ The authors found that the individual data were inadequate to determine the effect of dose or duration across the various studies.

\section{Hemorrhagic versus ischemic stroke risk}

Stroke events are generally characterized as either ischemic or hemorrhagic, with the majority of strokes being ischemic. ${ }^{1}$ 
As the mechanisms are different, one would infer that the risk of stroke with NSAIDs could be differentiated by subtype. Although NSAIDs have generally been predominantly associated with gastrointestinal bleeding, understanding of their effects on platelet function suggests that NSAID use could lead to an increased risk of hemorrhagic stroke, as well as ischemic stroke, through mediation of thrombosis. As the overall numbers of events have been limited within the prospective trials, the majority of stroke events quoted in various studies were not differentiated with respect to stroke subtypes. What little can be inferred of a potential for differential risk comes again from observational studies. A study by Johnsen et al found no increased risk of intracranial hemorrhage with NSAID exposure when subjects were stratified by gender, age, and history of hypertension. ${ }^{40}$ Additionally, two other studies found no association between either ischemic or hemorrhagic stroke, with any NSAID. ${ }^{30,41}$

\section{Risk by specific NSAID}

Although the overall literature suggests a possible association between the risk of stroke and use of NSAIDs, the risk, if any, of specific NSAID is additionally not definitive. Within the literature, there is some suggestion that COX-2 selectivity is proportional to an increased risk of stroke events. However, although some of the most highly COX-2-selective agents have been associated with increased stroke risk, ${ }^{32,34}$ the evidence with celecoxib is perhaps too divergent to draw any conclusions. Although the literature regarding MI events and celecoxib did not lead to its withdrawal, it did lead to a black box warning. The evidence behind celecoxib and stroke events is not nearly as supportive. As celecoxib is the only remaining COX-2-selective agent available on the United States market, it is important to critically analyze the data regarding any association. The previously mentioned study by Abraham et $\mathrm{al}^{31}$ found that the incidence of stroke increased proportionately with the degree of COX-2 selectivity. Additionally, the use of highly selective agents was significantly associated with stroke when compared with poorly selective agents. However, the use of moderately selective agents, such as celecoxib, was not significant compared with poorly selective agents. ${ }^{31}$ Several other observational studies that specifically studied celecoxib found no increased risk compared with that for non-NSAID users. ${ }^{32-34,42}$

Among the nonselective NSAIDs, the association with risk warrants further investigation, due to inconsistent data. Perhaps the best example of the lack of clarity is with regard to naproxen. A 2000 study of NSAID-associated gastrointestinal toxicity suggested a possible protective effect of naproxen compared with rofecoxib for MI, which was suggested to be due to its claimed near complete COX-1 inhibition at high dosage. ${ }^{43}$ However, although a few observational studies have not shown an association between risk of stroke and naproxen, ${ }^{30,33,34}$ others have shown a signal of harm with this agent. Naproxen has been associated with significant increases in blood pressure, which is a well-known risk factor for stroke. ${ }^{22}$ Two studies found that the use of naproxen was associated with elevated stroke risk, ${ }^{35,42}$ with one study finding that of the nonselective NSAIDs, naproxen actually was associated with a doubling of the risk for stroke. ${ }^{35}$ Adding further complication is the fact that several meta-analyses have used naproxen as the reference group for comparison with other NSAIDs, both selective and nonselective. ${ }^{37,44}$ Thus, although a definitive association between naproxen use and stroke events is not evident, based on its known effect on blood pressure, it would be reasonable to expect a possible association. Further study is warranted with regard to naproxen, particularly due to its wide availability and common use as an over-the-counter analgesic.

\section{Effect of duration of use}

Among the studies that have shown an association between NSAID use and stroke, the effect of duration of use is unclear. The larger study by Solomon et al notably found that the association between NSAID use and stroke risk became evident within the first 60 days and with rofecoxib, persisted for 3 years. ${ }^{33}$ In the study by Andersohn et al, ${ }^{32}$ it was found that the odds ratio for stroke risk tended to increase in a linear fashion for dose and duration of NSAID use. Additionally, a differential time risk may vary by NSAID type - with rofecoxib, the risk for stroke was elevated relatively early, at 3 months, but in the APPROVe and APC trials, the general cardiovascular risk was heightened for rofecoxib and celecoxib after at least 9 to 12 months of use. Thus, the current recommendations generally advise use of the lowest dose for the shortest duration needed, to avoid excess risk. ${ }^{7}$

\section{Special populations Elderly}

Elderly patients deserve particular mention as chronic use of NSAIDs increases with age, with an estimated up to $40 \%$ of individuals over age 65 years using either over-the-counter or prescribed NSAIDs. ${ }^{45,46}$ The elderly are known to have altered metabolism, and due to the increased risk of adverse gastrointestinal and cardiovascular side effects of NSAIDs, the use of NSAIDS in this population has been advised 
against. ${ }^{47}$ Additionally, the hypertensive effects of NSAIDs may be particularly prominent in the elderly. ${ }^{24,48,49}$ As the population continues to age and the need for NSAIDs increases, it is important to assess the risk in this special population. A few studies have specifically commented on or evaluated the effect of age, many of them focused on the military veteran population. A large study of over 300,000 veterans found that the incidence of stroke increased proportionately with degree of COX-2 selectivity. ${ }^{32}$ However, two other studies found no association of either hemorrhagic or ischemic stroke with any nonselective NSAID, in primarily elderly cohorts. ${ }^{30,50}$ Thus, the current evidence does not support an association between NSAID use and stroke risk in the elderly. Although further research within this population is needed, NSAID use should be avoided, when possible, in favor of non-NSAID analgesics.

\section{Women}

Although no specific data exist regarding the relative safety of NSAID use in women, any association may be of particular interest because of the recognized differential in stroke risk in men versus women. The risk of stroke over a lifetime is actually higher in women, ${ }^{51}$ and this risk is also modified by age, as the risk for women is lower at younger ages but increases with age, particularly above 75 years. ${ }^{52}$ Women are also more likely, in general, to use NSAIDs. ${ }^{34}$ The current guidelines ${ }^{53}$ recommend evaluating the risk of stroke as opposed to MI, in women, to determine need for aspirin for primary prevention, taking age into consideration as a modifying factor, based on evidence of a differential risk by gender. This would suggest that any association between NSAID use and stroke risk may also be affected by gender. However, as the overall data on this subject are limited, the specific data on women are relatively sparse. Although most studies did not stratify their results by gender, the large observational study by Andersohn et al did not find a specific association by gender, in over 450,000 patients. ${ }^{32}$ Two other studies in predominantly older women did find an association, with the highest risk of stroke associated with the use of rofecoxib. ${ }^{33,35}$ However, these studies also differed in their assessment of the risk with naproxen, with one finding a signal of harm for both rofecoxib and naproxen, ${ }^{35}$ while the other found an elevated risk with rofecoxib but a reduced risk with naproxen. ${ }^{33}$

\section{Summary and future directions}

Initial evidence from prospective trials demonstrating an increased incidence of adverse cardiovascular events led to the withdrawal of several selective COX-2 inhibitors. Although the evidence from the initial prospective trials suggested an association with coronary ischemic events, the evidence for a link between NSAIDs and stroke events remains a source of debate. As the evidence here has been presented, no definitive recommendations can be made regarding the use of NSAIDs with respect to stroke risk. However, alternative analgesics should be considered whenever possible, particularly in the elderly, and if NSAIDs are used, the duration should be as short as possible. Future studies should focus on further assessing the risk of specific vascular events, such as the risk of stroke with specific NSAIDs, both selective and nonselective. Two such trials may shed some light. One trial is designed to assess the safety of celecoxib compared with ibuprofen or naproxen, the two most commonly prescribe nonselective NSAIDs for the treatment of arthritis pain. Notably, patients with or at risk of developing cardiovascular disease will be recruited. ${ }^{54}$ An additional study will specifically compare the cardiovascular and gastrointestinal safety of celecoxib compared with nonselective NSAIDs, in patients over age 60 years, with either osteoarthritis or rheumatoid arthritis. ${ }^{55}$ Although neither trial has specified a separate stroke outcome, they should help further define an association between widely used NSAIDs, including celecoxib, and cardiovascular risk.

\section{Disclosure}

The authors report no conflicts of interest in this work.

\section{References}

1. Roger VL, Go AS, Lloyd-Jones DM, et al; American Heart Association Statistics Committee and Stroke Statistics Subcommittee. Heart disease and stroke statistics - 2012 update: a report from the American Heart Association. Circulation. 2012;125(1):e2-e220.

2. Singh G. Gastrointestinal complications of prescription and over-thecounter nonsteroidal anti-inflammatory drugs: a view from the ARAMIS database. Arthritis, Rheumatism, and Aging Medical Information System. Am J Ther. 2000;7(2):115-121.

3. Laine L. Approaches to nonsteroidal anti-inflammatory drug use in the high-risk patient. Gastroenterology. 2001;120(3):594-606.

4. Bresalier RS, Sandler RS, Quan H, et al; Adenomatous Polyp Prevention on Vioxx (APPROVe) Trial Investigators. Cardiovascular events associated with rofecoxib in a colorectal adenoma chemoprevention trial. N Engl J Med. 2005;352(11):1092-1102.

5. Greenberg JD, Fisher MC, Kremer J, et al; CORRONA Investigators. The COX-2 inhibitor market withdrawals and prescribing patterns by rheumatologists in patients with gastrointestinal and cardiovascular risk. Clin Exp Rheumatol. 2009;27(3):395-401.

6. Wilcox CM, Cryer B, Triadafilopoulos G. Patterns of use and public perception of over-the-counter pain relievers: focus on nonsteroidal antiinflammatory drugs. J Rheumatol. 2005;32(11):2218-2224.

7. Antman EM, Bennett JS, Daugherty A, Furberg C, Roberts H, Taubert KA; American Heart Association. Use of nonsteroidal antiinflammatory drugs: an update for clinicians: a scientific statement from the American Heart Association. Circulation. 2007;115(12):1634-1642.

8. Catella-Lawson F, Reilly MP, Kapoor SC, et al. Cyclooxygenase inhibitors and the antiplatelet effects of aspirin. $N$ Engl J Med. 2001;345(25):1809-1817. 
9. Warner TD, Mitchell JA. Cyclooxygenases: new forms, new inhibitors, and lessons from the clinic. FASEB J. 2004;18(7):790-804.

10. Cryer B, Feldman M. Cyclooxygenase-1 and cyclooxygenase-2 selectivity of widely used nonsteroidal anti-inflammatory drugs. Am J Med. 1998;104(5):413-421.

11. Smith SC, Benjamin EJ, Bonow RO, et al. AHA/ACCF secondary prevention and risk reduction therapy for patients with coronary and other atherosclerotic vascular disease: 2011 update: a guideline from the American Heart Association and American College of Cardiology Foundation endorsed by the World Heart Federation and the Preventive Cardiovascular Nurses Association. J Am Coll Cardiol. 2011;58(23):2432-2446

12. Fosslien E. Cardiovascular complications of non-steroidal antiinflammatory drugs. Ann Clin Lab Sci. 2005;35(4):347-385.

13. Catella-Lawson F, Crofford LJ. Cyclooxygenase inhibition and thrombogenicity. Am J Med. 2001;110 Suppl 3A:S28-S32.

14. Knights KM, Mangoni AA, Miners JO. Defining the COX inhibitor selectivity of NSAIDs: implications for understanding toxicity. Expert Rev Clin Pharmacol. 2010;3(6):769-776.

15. Kirkby NS, Lundberg MH, Harrington LS, et al. Cyclooxygenase-1, not cyclooxygenase-2, is responsible for physiological production of prostacyclin in the cardiovascular system. Proc Natl Acad Sci U SA. 2012;109(43):17597-17602.

16. Mangoni AA, Crilly MA, Knights KM. Cardiovascular toxicity of nonsteroidal anti-inflammatory drugs: moving beyond cyclooxygenase selectivity. Expert Rev Clin Pharmacol. 2011;4(3): 299-302.

17. Bowman TS, Gaziano JM, Kase CS, Sesso HD, Kurth T. Blood pressure measures and risk of total, ischemic, and hemorrhagic stroke in men Neurology. 2006;67(5):820-823.

18. ADAPT Research Group. Cardiovascular and cerebrovascular events in the randomized, controlled Alzheimer's Disease Anti-Inflammatory Prevention Trial (ADAPT). PLoS Clin Trials. 2006;1(7):e33.

19. Aisen PS, Schafer KA, Grundman M, et al; Alzheimer's Disease Cooperative Study. Effects of rofecoxib or naproxen vs placebo on Alzheimer disease progression: a randomized controlled trial. JAMA. 2003;289(21):2819-2826.

20. Collantes E, Curtis SP, Lee KW, et al; Etoricoxib Rheumatoid Arthritis Study Group. A multinational randomized, controlled, clinical trial of etoricoxib in the treatment of rheumatoid arthritis [ISRCTN25142273]. BMC Fam Pract. 2002;3:10.

21. Geusens P, Alten R, Rovensky J, et al. Efficacy, safety and tolerability of lumiracoxib in patients with rheumatoid arthritis. Int $J$ Clin Pract. 2004;58(11):1033-1041.

22. Pope JE, Anderson JJ, Felson DT. A meta-analysis of the effects of nonsteroidal anti-inflammatory drugs on blood pressure. Arch Intern Med. 1993;153(4):477-484.

23. Singh G, Fort JG, Goldstein JL, et al; SUCCESS-I Investigators. Celecoxib versus naproxen and diclofenac in osteoarthritis patients: SUCCESS-I Study. Am J Med. 2006;119(3):255-266.

24. Sowers JR, White WB, Pitt B, et al; Celecoxib Rofecoxib Efficacy and Safety in Comorbidities Evaluation Trial (CRESCENT) Investigators. The Effects of cyclooxygenase-2 inhibitors and nonsteroidal antiinflammatory therapy on 24-hour blood pressure in patients with hypertension, osteoarthritis, and type 2 diabetes mellitus. Arch Intern Med. 2005;165(2):161-168.

25. Johnson AG, Nguyen TV, Day RO. Do nonsteroidal anti-inflammatory drugs affect blood pressure? A meta-analysis. Ann Intern Med. 1994;121(4):289-300.

26. Bavry AA, Khaliq A, Gong Y, Handberg EM, Cooper-Dehoff RM, Pepine CJ. Harmful effects of NSAIDs among patients with hypertension and coronary artery disease. Am J Med. 2011;124(7): 614-620.

27. Solomon SD, McMurray JJ, Pfeffer MA, et al; Adenoma Prevention with Celecoxib (APC) Study Investigators. Cardiovascular risk associated with celecoxib in a clinical trial for colorectal adenoma prevention. N Engl J Med. 2005;352(11):1071-1080.
28. Silverstein FE, Faich G, Goldstein JL, et al. Gastrointestinal toxicity with celecoxib vs nonsteroidal anti-inflammatory drugs for osteoarthritis and rheumatoid arthritis: the CLASS study: A randomized controlled trial. Celecoxib Long-term Arthritis Safety Study. JAMA. 2000;284(10):1247-1255.

29. Bombardier C, Laine L, Reicin A, et al; VIGOR Study Group. Comparison of upper gastrointestinal toxicity of rofecoxib and naproxen in patients with rheumatoid arthritis. VIGOR Study Group. $N$ Engl $J$ Med. 2000;343(21):1520-1528.

30. Bak S, Andersen M, Tsiropoulos I, et al. Risk of stroke associated with nonsteroidal anti-inflammatory drugs: a nested case-control study. Stroke. 2003;34(2):379-386.

31. Abraham NS, El-Serag HB, Hartman C, Richardson P, Deswal A. Cyclooxygenase-2 selectivity of non-steroidal anti-inflammatory drugs and the risk of myocardial infarction and cerebrovascular accident. Aliment Pharmacol Ther. 2007;25(8):913-924.

32. Andersohn F, Schade R, Suissa S, Garbe E. Cyclooxygenase-2 selective nonsteroidal anti-inflammatory drugs and the risk of ischemic stroke: a nested case-control study. Stroke. 2006;37(7):1725-1730.

33. Solomon DH, Avorn J, Stürmer T, Glynn RJ, Mogun H, Schneeweiss S. Cardiovascular outcomes in new users of coxibs and nonsteroidal antiinflammatory drugs: high-risk subgroups and time course of risk. Arthritis Rheum. 2006;54(5):1378-1389.

34. Roumie CL, Mitchel EF, Kaltenbach L, Arbogast PG, Gideon P, Griffin MR. Nonaspirin NSAIDs, cyclooxygenase 2 inhibitors, and the risk for stroke. Stroke. 2008;39(7):2037-2045.

35. Haag MD, Bos MJ, Hofman A, Koudstaal PJ, Breteler MM, Stricker BH. Cyclooxygenase selectivity of nonsteroidal anti-inflammatory drugs and risk of stroke. Arch Intern Med. 2008;168(11):1219-1224.

36. Trelle S, Reichenbach S, Wandel S, et al. Cardiovascular safety of non-steroidal anti-inflammatory drugs: network meta-analysis. $B M J$. 2011;342:c7086.

37. Bhala N, Emberson J, Merhi A, et al; Coxib and traditional NSAID Trialists' (CNT) Collaboration. Vascular and upper gastrointestinal effects of non-steroidal anti-inflammatory drugs: meta-analyses of individual participant data from randomised trials. Lancet. 2013;382(9894):769-779.

38. Chen LC, Ashcroft DM. Do selective COX-2 inhibitors increase the risk of cerebrovascular events? A meta-analysis of randomized controlled trials. J Clin Pharm Ther. 2006;31(6):565-576.

39. Varas-Lorenzo C, Riera-Guardia N, Calingaert B, et al. Stroke risk and NSAIDs: a systematic review of observational studies. Pharmacoepidemiol Drug Saf. 2011;20(12):1225-1236.

40. Johnsen SP, Pedersen L, Friis S, et al. Nonaspirin nonsteroidal anti-inflammatory drugs and risk of hospitalization for intracerebral hemorrhage: a population-based case-control study. Stroke. 2003;34(2):387-391.

41. Choi NK, Park BJ, Jeong SW, Yu KH, Yoon BW. Nonaspirin nonsteroidal anti-inflammatory drugs and hemorrhagic stroke risk: the Acute Brain Bleeding Analysis study. Stroke. 2008;39(3):845-849.

42. Lee TA, Bartle B, Weiss KB. Impact of NSAIDS on mortality and the effect of preexisting coronary artery disease in US veterans. Am J Med. 2007;120(1):98. e9-e16.

43. Capone ML, Tacconelli S, Sciulli MG, et al. Clinical pharmacology of platelet, monocyte, and vascular cyclooxygenase inhibition by naproxen and low-dose aspirin in healthy subjects. Circulation. 2004;109(12):1468-1471.

44. Kearney PM, Baigent C, Godwin J, Halls H, Emberson JR, Patrono C. Do selective cyclo-oxygenase-2 inhibitors and traditional non-steroidal antiinflammatory drugs increase the risk of atherothrombosis? Meta-analysis of randomised trials. BMJ. 2006;332(7553):1302-1308.

45. Cullen DJ, Seager JM, Holmes S, et al. Pharmacoepidemiology of nonsteroidal anti-inflammatory drug use in Nottingham general practices. Aliment Pharmacol Ther. 2000;14(2):177-185.

46. Lanas A, Ferrandez A. Inappropriate prevention of NSAID-induced gastrointestinal events among long-term users in the elderly. Drugs Aging. 2007;24(2):121-131. 
47. American Geriatrics Society Panel on Pharmacological Management of Persistent Pain in Older Persons. Pharmacological management of persistent pain in older persons. J Am Geriatr Soc. 2009;57(8):1331-1346.

48. Whelton A, Fort JG, Puma JA, Normandin D, Bello AE, Verburg KM; SUCCESS VI Study Group. Cyclooxygenase-2 - specific inhibitors and cardiorenal function: a randomized, controlled trial of celecoxib and rofecoxib in older hypertensive osteoarthritis patients. Am J Ther. 2001;8(2):85-95.

49. Whelton A, White WB, Bello AE, Puma JA, Fort JG; SUCCESS-VII Investigators. Effects of celecoxib and rofecoxib on blood pressure and edema in patients $\geq 65$ years of age with systemic hypertension and osteoarthritis. Am J Cardiol. 2002;90(9):959-963.

50. Mangoni AA, Woodman RJ, Gilbert AL, Knights KM. Use of nonsteroidal anti-inflammatory drugs and risk of ischemic and hemorrhagic stroke in the Australian veteran community. Pharmacoepidemiol Drug Saf. 2010;19(5):490-498.

51. Seshadri S, Beiser A, Kelly-Hayes M, et al. The lifetime risk of stroke: estimates from the Framingham Study. Stroke. 2006;37(2):345-350.
52. Reeves MJ, Bushnell CD, Howard G, et al. Sex differences in stroke: epidemiology, clinical presentation, medical care, and outcomes. Lancet Neurol. 2008;7(10):915-926.

53. US Preventive Services Task Force. Aspirin for the prevention of cardiovascular disease: US Preventive Services Task Force recommendation statement. Ann Intern Med. 2009;150:396-404.

54. Becker MC, Wang TH, Wisniewski L, et al; PRECISION Investigators. Rationale, design, and governance of Prospective Randomized Evaluation of Celecoxib Integrated Safety versus Ibuprofen Or Naproxen (PRECISION), a cardiovascular end point trial of nonsteroidal antiinflammatory agents in patients with arthritis. Am Heart J. 2009;157(4):606-612.

55. Macdonald TM, Mackenzie IS, Wei L, Hawkey CJ, Ford I; SCOT study group collaborators. Methodology of a large prospective, randomised, open, blinded endpoint streamlined safety study of celecoxib versus traditional non-steroidal anti-inflammatory drugs in patients with osteoarthritis or rheumatoid arthritis: protocol of the standard care versus celecoxib outcome trial (SCOT). BMJ Open. 2013;3(1):e002295.
Vascular Health and Risk Management

\section{Publish your work in this journal}

Vascular Health and Risk Management is an international, peerreviewed journal of therapeutics and risk management, focusing on concise rapid reporting of clinical studies on the processes involved in the maintenance of vascular health; the monitoring, prevention and treatment of vascular disease and its sequelae; and the involvement of

\section{Dovepress}

metabolic disorders, particularly diabetes. This journal is indexed on PubMed Central and MedLine. The manuscript management system is completely online and includes a very quick and fair peer-review system, which is all easy to use. Visit http://www.dovepress.com/ testimonials.php to read real quotes from published authors. 